\title{
The Role of Late Dimercaptosuccinic Acid (DMSA) Scan and Renal Ultrasonography for Vesicoureteral Reflux in Older Children
}

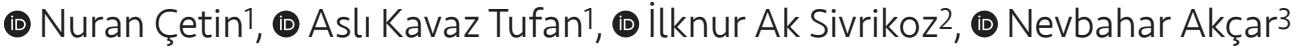 \\ ${ }^{1}$ Eskişehir Osmangazi University Faculty of Medicine, Department of Pediatric Nephrology, Eskişehir, Turkey \\ 2Eskişehir Osmangazi University Faculty of Medicine, Department of Nuclear Medicine, Eskişehir, Turkey \\ ${ }^{3}$ Eskişehir Osmangazi University Faculty of Medicine, Department of Radiology, Eskişehir, Turkey
}

\begin{abstract}
Aim: Vesicoureteral reflux (VUR) is an important risk factor for urinary tract infection (UTI). We aimed to investigate the relationships between VUR and ultrasound with late dimercaptosuccinic acid (DMSA) scan findings in children aged older than two years who had their first febrile UTI (FUTI).

Materials and Methods: Data from those patients with their first FUTI were retrospectively analyzed. A late DMSA scan was performed at least 6 months after an acute FUTI. The late DMSA scans were graded as mild (focal defect in uptake), moderate (uptake of renal radionuclide from 20 to 40\%), and severe (shrunken kidney with uptake less than 20\%). Micturating cystourethrography was performed at 3 to 6 weeks after the FUTI.

Results: The records of 220 patients ( 61 mild VUR, 60 severe VUR, 99 without VUR) were reviewed. An abnormal US was more common in those patients with VUR than those without VUR ( $p=0.009$ ). Abnormal US had a sensitivity of $79.34 \%$ for VUR and $81.67 \%$ for severe VUR. The negative predictive value of renal US for severe VUR was $91.13 \%$. The frequency of renal scarring was higher in those patients with VUR than for those without VUR (102/44, $p=0.001)$. A logistic regression analysis showed significant associations between abnormal US and VUR or severe VUR ( $p=0.019$ and $p=0.011$, respectively). Renal scarring had a sensitivity of $84.3 \%$ for VUR, and $91.67 \%$ for severe VUR.

Conclusion: Late DMSA scan findings can predict the presence and grade of VUR in older children who have their first FUTI. Normal renal US can predict the absence of severe VUR.

Keywords: Febrile urinary tract infection, late DMSA scan, older children, vesicoureteral reflux
\end{abstract}

\section{Introduction}

Urinary tract infections (UTI) are among the most common infections in childhood (1). The most common abnormality in children with UTI is vesicoureteral reflux (VUR). Children with VUR are at an increased risk of chronic kidney disease; therefore, it is important to determine the presence and grade of VUR in children with febrile UTI (FUTI) (2).

Diagnostic radiological studies in children with FUTI are controversial. The National Institute for Clinical Excellence guideline does not recommend micturating cystourethrography (MCUG) after a child's first UTI (3). In recent years, the emphasis has been on identifying 
patients who are at risk of recurrence of UTI and permanent renal damage rather than detecting VUR. This "top-down" approach implies that VUR has clinical significance only if it causes renal damage. A technetium 99 m-labeled dimercaptosuccinic acid (DMSA) scan is recommended to evaluate permanent renal damage (4).

In this study, we aimed to investigate the relationship between the presence of VUR and renal ultrasonography (US) with late DMSA scan findings in children older than two years who had their first FUTI.

\section{Materials and Methods}

The protocol for the present study was reviewed and approved by the Institutional Review Board of Eskişehir Osmangazi University Faculty of Medicine (approval number: 25403353-050.99-E.110593). In this retrospective study, the data of those patients with a first diagnosis of FUTI who were followed up in our Pediatric Nephrology Clinic between May 2015 and March 2019 were reviewed. Children with congenital anomalies of the kidney or urinary tract other than VUR, a history of FUTI and/or VUR diagnosis before the study and missing data and/or a follow-up period $<2$ years were excluded from the study. The patients with findings supporting congenital renal dysplasia, such as loss of corticomedullary differentiation and renal hyperechogenicity on US, and with unscarred kidneys with a differential function $<45 \%$ were not included.

Urine samples for culture were obtained from midstream urine. All of the patients underwent genital hygiene with soap and water in the laboratory before providing a urine sample. The diagnosis of FUTI was made on the basis of the presence of fever $>38{ }^{\circ} \mathrm{C}$ with at least 100,000 colonyforming units $/ \mathrm{mL}$ of a uropathogen cultured from the urine specimen and pyuria (leucocyte counts $\geq 5 /$ high-power field).

In our Pediatric Nephrology Department, all patients underwent a renal US within two weeks of their first FUTI. The DMSA scan was performed at least six months after the diagnosis of FUTI in all patients. MCUG was performed on those patients with serious illness, septicemia, poor urine flow, raised creatinine, failure to respond to treatment with suitable antibiotics within 48 hours or infection with nonE. coli organisms, hydronephrosis, and/or other findings suggestive of VUR on ultrasound. Only those patients with complete radiological examinations were included in this study.

Hydronephrosis was defined using the Society for Fetal Urology's grading system (5). Abnormal US was defined by hydronephrosis, hydroureter ( $\geq 7 \mathrm{~mm}$ ), parenchymal thinning, and dilatation of calyces.

Renal scarring was defined as a reduced or absent radionuclide uptake, a wedge-shaped defect, or the thinning or flattening of the renal outline. Reduced differential function (RDF) was defined as $<45 \%$. A kidney uptake of $45 \%-55 \%$ of the total renal activity was considered to be normal. The findings on DMSA scan were graded as mild (focal defect in uptake), moderate (uptake of renal radionuclide from $20 \%-40 \%$ ), and severe (shrunken kidney with uptake less than 20\%) (6). The DMSA scans were all evaluated by the same nuclear medicine specialist.

MCUG was performed at three to six weeks after the diagnosis of FUTI. VUR was graded according to the grading system of the International Reflux Study Committee. Grades 1-2 VUR were defined as mild VUR, while grades 3-5 VUR were defined as severe VUR. The pediatric radiologist and nuclear medicine specialist were unaware of the patients' clinical and laboratory findings.

\section{Statistical Analysis}

Statistical analyses were performed using SPSS version 11.0 (SPSS Inc, Chicago, IL). Values are expressed as a mean and standard deviation for continuous variables and as an interquartile range for qualitative variables. Qualitative variables were compared Ausing the chi-square test. A logistic regression analysis was performed to determine the association between the US findings and the DMSA scans in the presence of VUR. The sensitivity, specificity, positive predictive value, and negative predictive value (NPV) of US and late DMSA findings for VUR were calculated. A p-value $<0.05$ was considered significant.

\section{Results}

\section{Demographic and Imaging Features of the Study Group}

The records of 317 patients with a first febrile UTI were retrospectively reviewed in this study. The data of 97 patients who failed to meet the inclusion criteria were excluded from the study. The findings of the DMSA scan, renal US, and MCUG of the remaining 220 patients were analyzed. Figure 1 shows the flow of participant selection. The mean follow-up time was $3.9 \pm 1.37$ years (range 2.54 5.27 years). The mean age of the patients was $4.3 \pm 2.07$ years old (range 2-6.5 years). Of the 220 children, the female/ male ratio was 1.53:1 (133 girls and 87 boys).

Abnormal US was determined in 130 patients. Hydronephrosis was the most common finding $(n=86)$. 


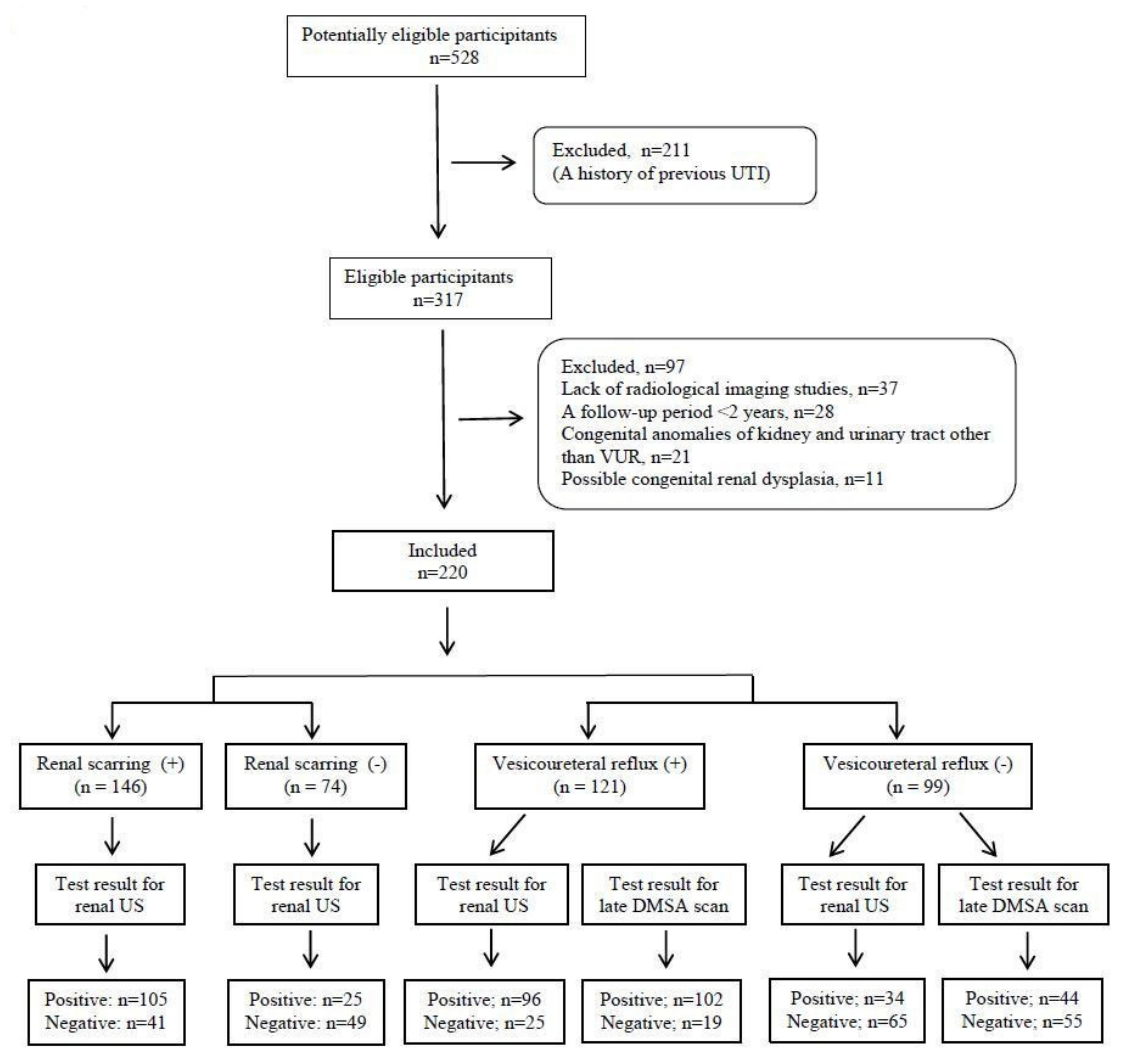

Figure 1. Participant selection diagram

VUR: Vesicoureteral reflux, UTI: Urinary tract infection, DMSA: Dimercaptosuccinic acid, US: Ultrasonography

Other US findings were as follows: hydroureter $(n=69)$, caliectasis $(n=60)$, and parenchymal thinning $(n=58)$. In 121 of the 220 patients, VUR was determined (61 patients showed mild VUR, and 60 showed severe VUR). US

The Relationship Between Late DMSA Scan and Renal

Renal scarring was determined in 146 patients (44 had mild scarring, 79 had moderate scarring, and 23 had severe scarring). The frequency of abnormal US was higher in those patients with scarring than those without scarring $(n=105$ and $n=25$, respectively; $p=0.011$ ).

Parenchymal thinning was shown in 58 patients, all of them with renal scarring. The frequency of hydronephrosis was higher in those patients with renal scarring than in those without renal scarring ( $n=68$ and $n=18$, respectively; $p=0.026)$. The frequency of parenchymal thinning was higher in those patients with severe scarring than in those patients with mild and moderate scarring $(n=15, n=16$, and $n=27$, respectively; $p=0.013$ ).

\section{The Renal Ultrasound and Late DMSA Scan Findings of the Patients with VUR}

The frequency of abnormal US was higher in those patients with VUR than in those without VUR ( $p=0.009)$. Those patients with VUR had higher frequencies of parenchymal thinning, hydronephrosis, and hydroureter $(p=0.000, p=0.022, p=0.000$, respectively; Table I). Those patients with severe VUR had higher frequencies of both hydronephrosis and parenchymal thinning than those patients with mild VUR ( $p=0.022$ and $p=0.000$, respectively; Table II). Twenty five (20.7\%) patients with VUR (14 with mild VUR, and 11 with severe VUR) had normal renal US.

Both moderate and severe scarring were common in the presence of VUR ( $p=0.000$ for both of them). Those patients with VUR had a higher frequency of RDF when compared to those patients without VUR ( $p=0.000$; Table I). A significant difference was not determined between those patients with mild and severe VUR in terms of the degree of renal scarring and RDF (detailed results are shown in Table II). 
Table I. The features of the patients with and without vesicoureteral reflux

\begin{tabular}{|l|l|l|l|}
\hline & $\begin{array}{l}\text { Vesicoureteral } \\
\text { reflux (+) } \\
\mathbf{n}(\%)\end{array}$ & $\begin{array}{l}\text { Vesicoureteral } \\
\text { reflux (-) } \\
\mathbf{n}(\%)\end{array}$ & p-value \\
\hline Gender (female) & $76(62.8)$ & $57(57.6)$ & 0.758 \\
\hline Abnormal US & $96(79.3)$ & $34(34.3)$ & 0.009 \\
\hline Hydronephrosis & $66(54.5)$ & $20(20.2)$ & 0.043 \\
\hline Caliectasis & $38(31.4)$ & $22(22.2)$ & 0.139 \\
\hline $\begin{array}{l}\text { Parenchymal } \\
\text { thinning }\end{array}$ & $48(39.7)$ & $10(10.1)$ & 0.001 \\
\hline Hydroureter & $61(50.4)$ & $8(8.1)$ & 0.000 \\
\hline Renal scarring & $102(84.3)$ & $44(44.4)$ & 0.001 \\
\hline Severe scarring & $18(14.9)$ & $5(5.1)$ & 0.000 \\
\hline Moderate scarring & $61(50.4)$ & $18(18.2)$ & 0.000 \\
\hline Mild scarring & $23(19)$ & $21(21.2)$ & 0.956 \\
\hline $\begin{array}{l}\text { Reduced } \\
\text { differential } \\
\text { function }\end{array}$ & $96(79.3)$ & $13(13.1)$ & 0.000 \\
\hline $\begin{array}{l}\text { Values were expressed as number and proportion. } \\
\text { A p-value <0.05 was considered significant } \\
\text { US: Ultrasonography }\end{array}$ & & \\
\hline
\end{tabular}

Table II. The features of the patients with mild and severe vesicoureteral reflux

\begin{tabular}{|l|l|l|l|}
\hline & $\begin{array}{l}\text { Mild } \\
\text { vesicoureteral } \\
\text { reflux (+) } \\
\mathbf{n}(\%)\end{array}$ & $\begin{array}{l}\text { Severe } \\
\text { vesicoureteral } \\
\text { reflux (+) } \\
\mathbf{n}(\%)\end{array}$ & p-value \\
\hline Gender (female) & $36(59)$ & $40(66.7)$ & 0.125 \\
\hline Abnormal US & $47(77.04)$ & $49(81.7)$ & 0.392 \\
\hline Hydronephrosis & $22(36.1)$ & $44(73.3)$ & 0.022 \\
\hline Caliectasis & $17(27.9)$ & $21(35)$ & 0.857 \\
\hline $\begin{array}{l}\text { Parenchymal } \\
\text { thinning }\end{array}$ & $8(13.2)$ & $40(66.7)$ & 0.000 \\
\hline Hydroureter & $17(27.9)$ & $44(73.3)$ & 0.029 \\
\hline Renal scarring & $47(77.04)$ & $55(91.7)$ & 0.046 \\
\hline Severe scarring & $9(14.8)$ & $9(15)$ & 0.911 \\
\hline Moderate scarring & $29(47.5)$ & $32(53.3)$ & 0.548 \\
\hline Mild scarring & $9(14.8)$ & $14(23.3)$ & 0.106 \\
\hline $\begin{array}{l}\text { Reduced } \\
\text { differential } \\
\text { function }\end{array}$ & $44(72.1)$ & $52(86.7)$ & 0.094 \\
\hline $\begin{array}{l}\text { Values were expressed as number and proportion. } \\
\text { A p-value <0.05 was considered significant } \\
\text { US: Ultrasonography }\end{array}$ & & \\
\hline
\end{tabular}

The Relationships Between Renal US, Late DMSA Scan, and the Presence/Grade of VUR

A logistic regression analysis showed a significant association between abnormal US and VUR or severe VUR $(p=0.019$ and $p=0.011$, respectively). Parenchymal thinning was associated with both VUR and severe VUR ( $p=0.038$ and $p=0.010$, respectively). Renal scarring had a predictive value for VUR $(p=0.005)$. While severe and moderate scarring were associated with the presence of VUR, there was no significant relationship between severe VUR and the degree of renal scarring. There was a significant association between RDF and VUR but not severe VUR ( $p=0.015$ and $p=0.242$, respectively). Detailed results of the logistic regression analysis are shown in Table III.

The true and false positive/negative values and diagnostic accuracy of late DMSA scan and US to detect VUR and severe VUR are shown in Tables IV and V. Abnormal ultrasound had a sensitivity of $79.34 \%$ for VUR and $81.67 \%$ for severe VUR. The specificity of abnormal ultrasound was $65.66 \%$ for VUR and $70.63 \%$ for severe VUR. The NPV of abnormal US was $91.13 \%$ for severe VUR. Renal scarring on late DMSA scan had a sensitivity of $84.3 \%$ for VUR and 91.67 $\%$ for severe VUR. The NPV of renal scarring was $73.68 \%$ for severe VUR.

\section{Discussion}

The results of our study showed that parenchymal thinning and hydroureter were significant indicators for both the presence and degree of VUR in older children who had their first FUTI. There was a significant association between the presence of VUR and renal scarring and RDF on late DMSA scan. However, no relationship was found between the degree of renal scarring and different grades of VUR.

VUR is considered to be one of the most common urological anomalies that can predispose children to UTI. The incidence of VUR varies between $0.5 \%$ and $3 \%$ in healthy children, but the frequency of VUR rises to $30-64 \%$ in children with UTI $(7,8)$. In our study, VUR was detected in $55 \%$ of patients who had their first FUTI. This result supports the need for more determining indicators to perform MCUG in children during their first FUTI so as to not miss a diagnosis of VUR.

An acute-phase DMSA scan has an important role in detecting parenchymal infection during active infection. Previous studies have shown that while the frequency of abnormal DMSA scan ranged from $51 \%$ to $73 \%$ during the acute period of FUTI, the frequency of permanent scarring 
Table III. Logistic regression analysis showing factors associated with presence of vesicoureteral reflux and severe vesicoureteral reflux

\begin{tabular}{|c|c|c|c|c|c|c|}
\hline & \multicolumn{3}{|c|}{ Vesicoureteral reflux } & \multicolumn{3}{|c|}{ Severe vesicoureteral reflux } \\
\hline & OR & $95 \% \mathrm{Cl}$ & p-value & OR & $95 \% \mathrm{Cl}$ & p-value \\
\hline Female gender & 0.869 & $0.217-1,036$ & 0.981 & 0.346 & 0.017-0.936 & 0.851 \\
\hline Abnormal US & 1,549 & $1,215-5,541$ & 0.019 & 1,417 & $1,153-6,439$ & 0.011 \\
\hline Hydronephrosis & 0.816 & $0.882-5,799$ & 0.089 & 1,828 & $1,212-31,937$ & 0.028 \\
\hline Caliectasis & 0.522 & $0.575-4,936$ & 0.341 & 0.191 & $0.156-2,650$ & 0.459 \\
\hline Parenchymal thinning & 1,130 & $1,058-7,415$ & 0.038 & 2,175 & $1,692-45,761$ & 0.010 \\
\hline Hydroureter & 2,350 & $1,265-6,796$ & 0.014 & 3,689 & $1,551-5,271$ & 0.003 \\
\hline Renal scarring & 2,317 & $1,624-9,174$ & 0.005 & 1,216 & $1,108-1,471$ & 0.043 \\
\hline Severe scarring & 1,416 & $1,216-4,480$ & 0.038 & 0.362 & $0.218-1,019$ & 0.837 \\
\hline Moderate scarring & 1,248 & $1,116-2,635$ & 0.041 & 0.503 & $0.259-0.925$ & 0.757 \\
\hline Mild scarring & 0.415 & $0.172-1,109$ & 0.691 & 0.215 & $0.127-0.815$ & 0.947 \\
\hline Reduced differential function & 1,420 & $1,324-12,936$ & 0.015 & 0.386 & $0.025-2,550$ & 0.242 \\
\hline
\end{tabular}

Table IV. The true and false positive/negative values for late DMSA scan and ultrasound to detect VUR and severe VUR

\begin{tabular}{|l|l|l|l|l|}
\hline Test result & VUR (+) & VUR (-) & $\begin{array}{l}\text { Severe } \\
\text { VUR (+) }\end{array}$ & $\begin{array}{l}\text { Mild } \\
\text { VUR }\end{array}$ \\
\hline Renal scarring & 102 & 44 & 55 & 47 \\
\hline Positive & 19 & 55 & 5 & 14 \\
\hline Negative & 121 & 99 & 60 & 61 \\
\hline $\begin{array}{l}\text { Abnormal renal } \\
\text { ultrasound }\end{array}$ & 96 & 34 & 49 & 47 \\
\hline Positive & 25 & 65 & 11 & 14 \\
\hline Negative & 121 & 99 & 60 & 61 \\
\hline VUR: Vesicoureteral reflux, DMSA: Dimercaptosuccinic acid \\
\hline
\end{tabular}

on the late DMSA scan was only $9.5 \%-11.9 \%(9,10)$. Studies have also reported a higher prevalence of permanent renal damage after acute pyelonephritis (11). Orellana et al. (12) showed that children older than one year had a higher frequency of renal scarring than infants. The frequency of permanent renal scarring was quite high $(66.4 \%)$ in our patients who had their first FUTI. The high frequency of renal scarring might be due to the fact that we included older children in our study. In addition, differences such as type of imaging study, age at diagnosis, clinical presentation, and the distribution of the severity of VUR could affect the prevalence of renal scarring.

There is little consensus and little data on imaging after FUTI in older children (13). Hitzel et al. (14) suggest that MCUG is not necessary in children who have a normal US and DMSA scan. The revised guidelines by the Indian Pediatric Nephrology Group recommend DMSA scan as the
Table V. Diagnostic accuracy of renal bladder ultrasound and late DMSA scan to detect vesicoureteral reflux and severe vesicoureteral reflux

\begin{tabular}{|l|l|l|}
\hline & \multicolumn{1}{|l|}{$\begin{array}{l}\text { Vesicoureteral } \\
\text { reflux }\end{array}$} & $\begin{array}{l}\text { Severe } \\
\text { vesicoureteral } \\
\text { reflux }\end{array}$ \\
\hline Abnormal ultrasound & $79.34(71.03-86.16)$ & $81.67(69.56-90.48)$ \\
\hline Sensitivity (\%) & $65.66(55.44-74.91)$ & $70.63(62.92-77.55)$ \\
\hline Specificity (\%) & $73.85(67.94-79)$ & $51.04(44.35-57.69)$ \\
\hline + Predictive value (\%) & $72.22(64.07-79.13)$ & $91.13(85.65-94.65)$ \\
\hline - Predictive value (\%) & Renal scarring on late DMSA scan & $91.67(81.61-97.24)$ \\
\hline Sensitivity (\%) & $84.3(76.57-90.27)$ & $22.95(13.15-35.53)$ \\
\hline Specificity (\%) & $55.56(45.22-65.55)$ & $53.92(50.01-57.79)$ \\
\hline + Predictive value (\%) & $69.86(64.74-74.54)$ & $73.68(51.82-87.94)$ \\
\hline - Predictive value (\%) & $74.32(64.89-81.93)$ & \\
\hline DMSA: Dimercaptosuccinic acid & \\
\hline
\end{tabular}

first approach (followed by VCUG if positive) in children between the ages of one and five years after their first UTI (15). Doğan et al. (16) found that abnormal DMSA scan findings had a sensitivity of $83.87 \%$ for VUR in 32 children older than two years with recurrent UTI. However, their study included patients with urological abnormalities other than VUR, as well as children with bladder and/or bowel dysfunction (16). Balestracci et al. (17) investigated the predictive role of late DMSA scan for high-grade VUR in 122 children aged between 3 and 18 years with FUTI. They also included patients with FUTI which occurred before the 
first year of life. Of their patients, 57.4\% had an abnormal late DMSA scan. Abnormal late DMSA scan was associated with both VUR and high-grade VUR (17). We determined a sensitivity of $84.3 \%$ of the abnormal late DMSA scan for the presence of VUR. Our study included DMSA scan findings of patients with their first FUTI. We did not include patients with urological abnormalities other than VUR, or patients with bladder and/or bowel dysfunction or a history of FUTI before the study. Our results showed that abnormal late DMSA scan could predict the presence of VUR in older children who had their first FUTI.

Researchers have drawn different conclusions about the relationship between abnormal DMSA scan and severe VUR. Silva et al. (18) reported that severe VUR was associated with all subtypes of renal damage. In their study, the median age at diagnosis of the first UTI was 8.9 months, and the median age at diagnosis of VUR was 19 months. Interestingly, Jang et al. (19) showed that abnormal DMSA scan was more common in the presence of severe VUR in patients less than 1 year old. However, they could not show the same relationship in patients over 1 year old (19). Lee et al. (20) did not find a significant difference in the prevalence of renal scarring between the different grades of VUR in children between 0 and 5 years of age. In our study, late DMSA scan had a sensitivity of $91.67 \%$ for severe VUR. However, we did not identify a significant association between severe VUR and the degree of renal scarring.

The literature contains conflicting conclusions about the predictive value of renal US for VUR. Several studies found no significant association between US findings and the presence of VUR (21). Conversely, Darge (22) reported that cortical thinning, small kidneys, and cortical hyperechogenicity were associated with the presence of VUR. Doğan et al. (16) reported that renal US had a sensitivity of $75 \%$ for the presence of VUR. In the study by Balestracci et al. (17) of the 69 patients with normal renal US, 32 (46.3\%) had VUR. In our study, abnormal US had a sensitivity of $79.34 \%$ for VUR; nevertheless, one-fifth of our patients with VUR had no abnormal US findings. Our results indicated that older children who had their first FUTI could have VUR even if renal US is normal.

There are also different opinions concerning the ability of US to detect the grade of VUR. Bayram et al. (23) reported that the frequency of abnormal US was higher in patients with grades 4-5 of VUR (18). Another study showed renal US had a sensitivity of $63 \%-86 \%$ in the diagnosis of severe VUR $(19,24)$. Our results revealed that the sensitivity of abnormal renal US was $81.67 \%$, and NPV was $91.13 \%$ for severe VUR. Based on the results of our study, normal renal US could indicate the absence of severe VUR in older children who have their first FUTI.

VUR is thought to be the most important risk factor for the development of renal scarring. However, recently, it has been suggested that UTI is more closely related to the development of permanent scarring than VUR (25). In our study, 44 patients had scarring but did not have VUR. This result might indicate that factors other than VUR, such as the severity of the inflammatory reaction, differences in the immunological system, and microbiological virulence factors, could play a role in the development of renal scarring.

Although renal US provides information about anatomic disorders of the kidney and urinary tract, several studies have shown that US is not a good determinant of renal scarring after the first UTI. Bush et al. (26) demonstrated that about one-fifth of children diagnosed with FUTI had renal scarring despite normal renal US. The false negative rate of renal US was $23 \%$ in children aged two years and older (21). Inversely, Merguerian et al. (27) found a significant correlation between renal US findings and diffuse renal scar, although there was a weak correlation between focal scar and renal US (22). In our study, about a third of patients with renal scarring had normal renal US. Accordingly, normal renal US cannot exclude the presence of renal scarring on late DMSA scan.

\section{Study Limitations}

This study has several limitations. First, this is a retrospective study with a small sample size. Second, our study could not distinguish whether DMSA scan abnormalities were congenital or acquired in patients without VUR. DMSA defects in these patients might be caused by congenital dysplasia, rather than UTI or VUR. Third, the reason for the high frequency of renal scarring might be related to possible previously undiagnosed infections. However, since this study was based on recorded medical data, we could not ascertain whether the symptoms of UTI had been present previously.

\section{Conclusion}

Our results showed that late DMSA scan findings were able predict the presence and grade of VUR in older children who had their first FUTI, and normal renal US was able predict the absence of severe VUR. However, older children who had their first FUTI should be investigated in terms of VUR after acute infection, even if renal scarring and abnormal renal US is not detected. 


\section{Ethics}

Ethics Committee Approval: The protocol for the present study was reviewed and approved by the Institutional Review Board of Eskişehir Osmangazi University Faculty of Medicine (approval number: 25403353-050.99-E.110593).

Informed Consent: Retrospective study.

Peer-review: Externally peer-reviewed.

\section{Authorship Contributions}

Concept: N.Ç., Design: N.Ç., A.K.T., Data Collection or Processing: N.Ç., Analysis or Interpretation: N.Ç., I.A.S., N.A., Writing: N.Ç., A.K.T., I.A.S., N.A.

Conflict of Interest: No conflict of interest was declared by the authors.

Financial Disclosure: The authors declared that this study received no financial support.

\section{References}

1. Shaikh N, Morone NE, Bost JE, Farrell MH. Prevalence of urinary tract infection in childhood: a meta-analysis. Pediatr Infect Dis I 2008; 27:302-8.

2. Downs SM. Technical report: urinary tract infections in febrile infants and young children. Pediatrics 1999; 103:e54. doi: 10.1542/peds.103.4.e54.

3. Mori R, Lakhanpaul M, Verrier-Jones K. Diagnosis and management of urinary tract infection in children: summary of NICE guidance. BMJ 2007; 335:395-7.

4. Riccabona M, Avni FE, Blickman JG, et al. Imaging recommendations in paediatric uroradiology: minutes of the ESPR workgroup session on urinary tract infection, fetal hydronephrosis, urinary tract ultrasonography and voiding cystourethrography, Barcelona, Spain, June 2007. Pediatr Radiol 2008; 38:138-45.

5. Nguyen HT, Herndon CD, Cooper C, et al. The Society for Fetal Urology consensus statement on the evaluation and management of antenatal hydronephrosis. J Pediatr Urol 2010; 6:212-31.

6. Polito C, La Manna A, Rambaldi PF, Nappi B, Mansi L, Di Toro R. High incidence of a generally small kidney and primary vesicoureteral reflux. J Urol 2000; 164:479-82.

7. MacKenzie JR, Fowler K, Hollman AS, et al. The value of ultrasound in the child with an acute urinary tract infection. Br J Urol 1994; 74:240-4.

8. Herz D, Merguerian P, McQuiston L, et al. 5-year prospective results of dimercapto-succinic acid imaging in children with febrile urinary tract infection: proof that the top-down approach works. J Urol 2010; 184(Suppl 4):1703-9.

9. RIVUR Trial Investigators, Hoberman A, Greenfield SP, et al. Antimicrobial prophylaxisfor children with vesicoureteral reflux. N Engl I Med 2014; 370:2367-76.

10. Shaikh N, Mattoo TK, Keren R, et al. Early antibiotic treatment for pediatric febrile urinary tract infection and renal scarring. JAMA Pediatr 2016; 170:848-54.
11. Lin KY, Chiu NT, Chen MJ, et al. Acute pyelonephritis and sequelae of renal scar in pediatric first febrile urinary tract infection. Pediatr Nephrol 2003; 18:362-5.

12. Orellana $\mathrm{P}$, Baquedano $\mathrm{P}$, Rangarajan $\mathrm{V}$, et al. Relationship between acute pyelonephritis, renal scarring, and vesicoureteral reflux. Results of a coordinated research project. Pediatr Nephrol 2004; 19:1122-6.

13. Kurtz MP, Chow JS, Johnson EK, Rosoklija I, Logvinenko T, Nelson CP. Imaging after urinary tract infection in older children and adolescents. J Urol 2015; 193(Suppl 5):1778-82.

14. Hitzel A, Liard A, Véra P, Manrique A, Ménard JF, Dacher JN. Color and power Doppler sonography versus DMSA scintigraphy in acute pyelonephritis and in prediction of renal scarring. I Nucl Med 2002; 43:27-32.

15. Indian Society of Pediatric Nephrology, Vijayakumar $M$, Kanitkar M, Nammalwar BR, Bagga A. Revised statement on management of urinary tract infections. Indian Pediatr 2011; 48:709-17.

16. Doğan ÇS, Koyun NS, Aksoy GK, Çekiç B, Savaş M, Çomak E. Delayed diagnosis of primary vesicoureteral reflux in children with recurrent urinary tract infections: diagnostic approach and renal outcomes. Turk J Urol 2018; 44:498-502.

17. Balestracci A, Montecuco M, Serviddio C, et al. Role of late DMSA renal scan in detecting high-grade vesicoureteral reflux. Indian / Pediatr 2019; 86:784-9.

18. Silva JM, Diniz JS, Lima EM, et al. Independent risk factors for renal damage in a series of primary vesicoureteral reflux: a multivariate analysis. Nephrology (Carlton) 2009; 14:198-204.

19. Jang $\mathrm{HC}$, Lee $\mathrm{KH}$, Park IS. Primary vesico-ureteral reflux: comparison of factors between infants and children. Korean I Urol 2011; 52:206-9.

20. Lee $J \mathrm{H}$, Son $\mathrm{CH}$, Lee MS, Park YS. Vesicoureteral reflux increases the risk of renal scars: a study of unilateral reflux. Pediatr Nephrol 2006; 21:1281-4.

21. Montini G, Zucchetta P, Tomasi L, et al. Value of imaging studies after a first febrile urinary tract infection in young children: data from Italian renal infection study 1. Pediatrics 2009; 123:e23946. doi: 10.1542 /peds.2008-1003

22. Darge K. Diagnosis of vesicoureteral reflux with ultrasonography. Pediatr Nephrol 2002; 17:52-60.

23. Bayram MT, Kavukcu S, Alaygut D, Soylu A, Cakmakci H. Place of ultrasonography in predicting vesicoureteral reflux in patients with mild renal scarring. Urology 2014; 83:904-8.

24. Tsai JD, Huang CT, Lin PY, et al. Screening high-grade vesicoureteral reflux in young infants with a febrile urinary tract infection. Pediatr Nephrol 2012; 27:955-63.

25. Prasad MM, Cheng EY. Radiographic evaluation of children with febrile urinary tract infection: bottom-up, top-down, or none of the above? Adv Urol 2012: 716-39.

26. Bush NC, Keays M, Adams C, et al. Renal damage detected by DMSA, despite normal renal ultrasound, in children with febrile UTI. J Pediatr Urol 2015; 11:126.e1-7.

27. Merguerian PA, Jamal MA, Agarwal SK, et al. Utility of SPECT DMSA renal scanning in the evaluation of children with primary vesicoureteral reflux. Urology 1999; 53:1024-8. 